\title{
Is Porphyromonas gingivalis involved in Parkinson's disease?
}

\author{
Ingar Olsen ${ }^{1}$ (D) Douglas B. Kell ${ }^{2,3} \cdot$ Etheresia Pretorius $^{3}$
}

Received: 5 March 2020 / Accepted: 8 June 2020 / Published online: 21 June 2020

(C) The Author(s) 2020

\begin{abstract}
Porphyromonas gingivalis, a major subgingival plaque bacterium in periodontitis, has recently attracted much attention as a possible microbial driver in Alzheimer's disease. In the present paper, another common neuroinflammatory disease, Parkinson's disease (PD), is discussed. A recent study found major virulence factors of $P$. gingivalis such as gingipain R1 (RgpA) and lipopolysaccharide in the blood circulation of a PD population. The current review reveals how features such as systemic inflammation, hypercoagulation, presence of amyloid fibrin(ogen) in plasma, and marked ultrastructural changes in platelets, probably induced by $P$. gingivalis, may affect the development of PD. Several other clinical studies have also demonstrated an association between periodontitis and PD. Even if the risk of periodontal diseases causing neurological disorders needs to be better substantiated, that should not keep us from trying to prevent them by performing careful daily dental hygiene.
\end{abstract}

Keywords Systemic inflammation $\cdot$ Cytokines $\cdot$ Hypercoagulation $\cdot$ Amyloid formation $\cdot$ Gingipains $\cdot$ LPS

\section{Introduction}

Periodontitis is a collection of diseases where microorganisms cause destruction of the tooth-supporting structures through poorly controlled inflammatory responses. A world workshop held in 2017 distinguished periodontitis in three clinical forms: necrotizing periodontitis, periodontitis as a manifestation of systemic disease, and the forms of the disease previously recognized as chronic or aggressive, now grouped under a single category, periodontitis [1].

The oral cavity contains up to 1000 different bacteria localized in different niches, each with a characteristic microbiota [2]. A homeostatic balance exists most of the time between host and oral microbes, but this can be tipped towards disease. Traditional culture-based methods emphasized the anaerobic Gram-negative rods Porphyromonas gingivalis, Tannerella forsythia, and Treponema denticola (the red complex) as the

Ingar Olsen

ingar.olsen@odont.uio.no

1 Department of Oral Biology, Faculty of Dentistry, University of Oslo, POB 1052 Blindern, 0316 Oslo, Norway

2 Department of Biochemistry, Institute of Integrative Biology, Faculty of Health and Life Sciences, University of Liverpool, Liverpool, UK

3 Department of Physiological Sciences, Faculty of Science, Stellenbosch University, Stellenbosch, South Africa major pathogens of periodontitis [3]. Culture-independent methods have extended this list to comprise both Grampositive and Gram-negative bacteria (reviewed by Lamont et al. [4]). Among all these species $P$. gingivalis has emerged as a keystone bacterium in periodontitis $[5,6]$.

In periodontitis, polymicrobial communities initiate a dysregulated host response through polymicrobial synergy and dysbiosis [7]. As a keystone pathogen, $P$. gingivalis increases the nososymbiosity of subgingival microbial communities and drives periodontitis pathogenicity, remarkably, even at a low abundance [5]. In this interplay, inflammation is an important ecological factor that can stimulate outgrowth of periodontitis-associated microorganisms by tissue destruction releasing nutrients (e.g., degraded collagen, haeme-containing complexes, amino acids, and iron). The nutrients are transferred to the subgingival bacteria, to which $P$. gingivalis belongs, by the inflammatory exudate of the gingival crevicular fluid. Also increase in genes important for the pathogenesis of periodontitis such as proteolysis-related genes and genes for peptide transport and acquisition of iron and genes for synthesis of lipopolysaccharides (LPSs) have been detected. These genes elevate the proinflammatory potential of the microbial community [8]. Remodeling of the original biofilm into a dysbiotic one increases the capability of the biofilm to release proinflammatory cytokines from host cells [9]. P. gingivalis also uncouples inflammation from the bactericidal activity of leukocytes [5]. This subversive action disrupts the microbial homeostasis and contributes to development of a dysbiotic 
microbiota and periodontitis. Furthermore, $P$. gingivalis selectively suppresses IL-8 and Thelper 1 cell-biasing chemokines (CXCL9, CXCL10, and CXCL11) [10]. By manipulating host immunity and keeping bactericidal and inflammatory activities apart, $P$. gingivalis increases the adaptive fitness of the entire microbial community [4].

\section{Neuroinflammation and Parkinson's disease}

Parkinson's disease (PD) is characterized by a number of pathologies (Fig. 1) from misfolding $\alpha$-synuclein ( $\alpha$ Syn) to neuroinflammation, mitochondrial dysfunction, neurotransmitterdriven changes in neuronal networks of the brain, and affection of the brain-gut axis (for a review, see Adams et al. [11]). The present review deals with the possible role of $P$. gingivalis as a driving force in developing $\mathrm{PD}$, focusing on recent information.

Neuroinflammation is a characteristic feature of PD [12-14]. There are higher levels of inflammatory cytokines in the brain of PD patients than in controls, and inflammation seems to be an important factor in the process of neurodegeneration [15]. Furthermore, chronic inflammation, causing dysregulation of circulating inflammatory molecules, and an innate immune response seem to be important features in PD [16]. Both peripheral and brain inflammation contribute to initiation and progression of the neurodegeneration (for a review, see Adams et al. [11]). Increased levels of circulating cytokines such as IL-1 $\beta$, IL-2, IL-10, IL-6, IL-4, TNF- $\alpha$, Creactive protein, RANTES (member of the interleukin- 8 superfamily of cytokines), and interferon-gamma (INF- $\gamma$ ) have been found in PD [11, 17, 18], accompanied by oxidative stress [19]. In addition to significantly increased levels of circulating proinflammatory cytokines, $\mathrm{PD}$ was characterized by hypercoagulability (demonstrated by thromboelastograpy (TEG), confocal and electron microscopy) and an abnormal clotting potential [11]. Blood platelets showed marked ultrastructural changes and amyloid deposition was detected in the plasma [11].

\section{Porphyromonas gingivalis and Parkinson's disease}

The reason for the chronic neuroinflammation in PD is unclear. Attention has been paid to dormant microbes, which can shed inflammagens such as LPS and lipoteichoic acid [20, 21]. These are ligands for Toll-like receptor 4 (TLR4) that can activate inflammation [22]. Bacterial inflammagens that recently attracted the attention in PD were proteases from the periodontal pathogen P. gingivalis [11] (Fig. 2). As mentioned, this bacterium is considered a keystone pathogen in periodontitis causing microbial dysbiosis typical of the pathogenesis. $P$. gingivalis may also be involved in other systemic inflammatory diseases such as type 2 diabetes mellitus, Alzheimer's disease, rheumatoid arthritis, and cardiovascular disease (for reviews, see $[11,23]$ ).

$P$. gingivalis has a number of ways to reach the brain from the infected periodontal pocket [24] and has been detected in the brain of animals and humans with Alzheimer's disease $[25,26]$ where its LPS and cysteine proteases known as gingipains have been implicated in disease causation. As mentioned, proteolysis is important for this bacterium to obtain nutrition through breakdown of proteins. Gingipains are also important for degradation of antibacterial peptides [27] and for the bacterium to evade the complement system [28, 29].

\section{Hypercoagulation, proinflammatory cytokines, and plasma amyloid formation in Parkinson's disease}

In the study by Adams et al. [11], PD individuals were found to have a dysregulated profile of inflammatory biomarkers. Their whole blood was hypercoagulable with hyperactivated blood platelets and contained fibrin(ogen) with amyloid features. An inter-linked relationship between hypercoagulability, presence of inflammatory molecules, and activation of platelets was suggested. Furthermore, platelet pathology (hyperactivation, spreading, aggregation, or clumping), anomalous fibrin(ogen) protein structure, and red blood cell eryptosis were detected and interpreted as reflecting a systemic inflammation. The significantly upregulated cytokines (IL- $1 \alpha$, IL$1 \beta$, IL-17A, and TNF- $\alpha$ ) detected were related to the change in the ultrastructure of hyperactivated platelets. Fibrinogen levels have also previously been found to be higher in PD patients than in healthy controls [30, 31]. In the study by Adams et al. [11], fibrinogen in clots polymerized into an increased number of $\beta$-sheets that reflected formation of an amyloid protein structure. These protein changes were visualized using fluorescent amyloid markers called Amytrackers. Such protein changes might affect the anomalous clot formation and emphasized the systemic nature of PD. Fibrin(ogen) that showed an amyloid protein structure (as shown by fluorescent amyloid markers including Amytrackers and thioflavin $\mathrm{T}$ ) has also been detected in other diseases with systemic inflammation such as type 2 diabetes [32, 33] and Alzheimer's disease [34] where it is a major feature.

\section{Gingipains and lipopolysaccharide in Parkinson's disease}

The gingipains Rgp and $\mathrm{Kgp}$ of $P$. gingivalis have been found to increase the thrombin time compared with controls [35]. Also other coagulation factors can be activated by gingipains 


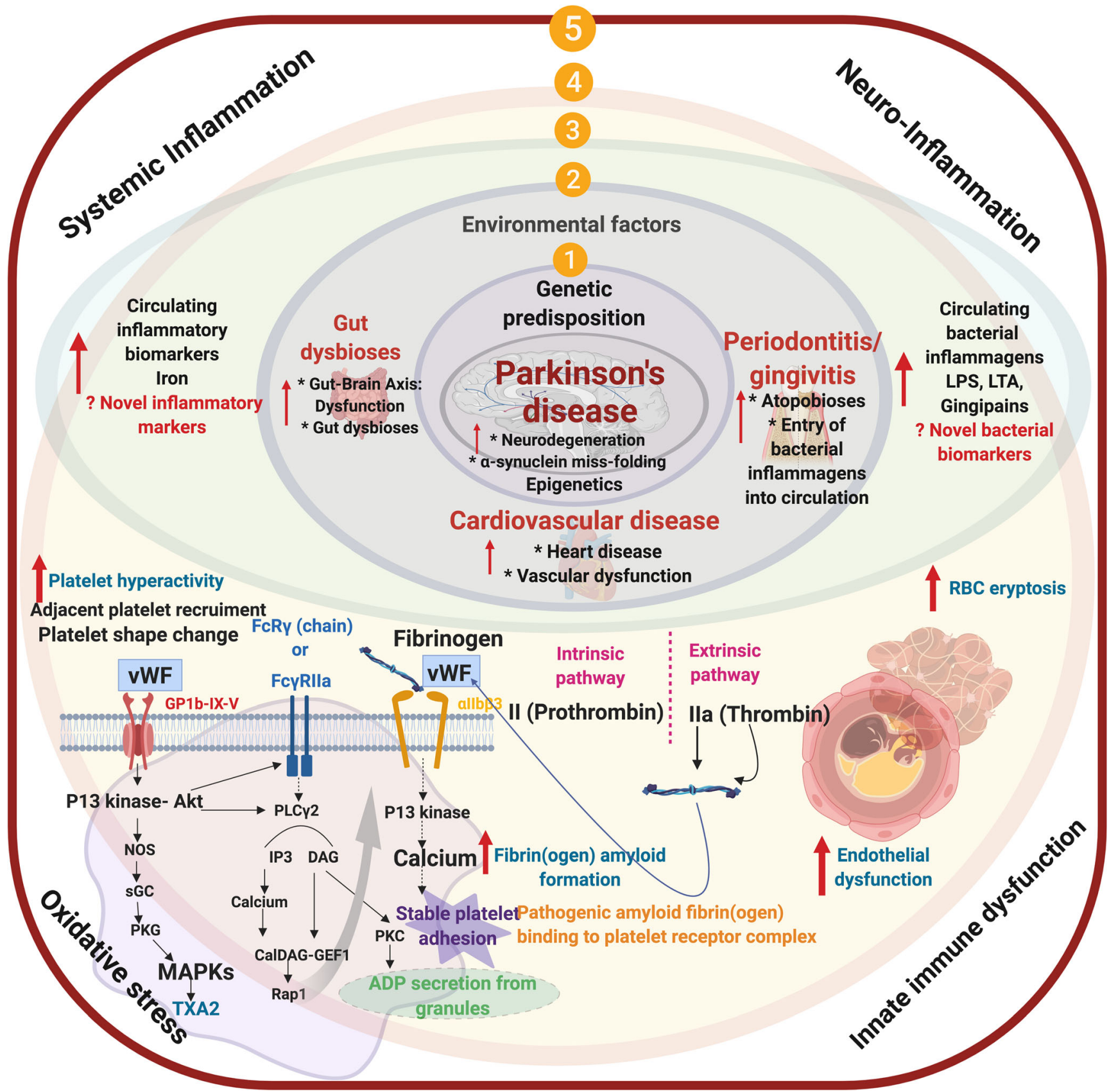

Fig. 1 (1) Genetic/epigenetic predisposition and (2) environmental factors that culminate in an individual that will have gut dysbiosis, and/or gingivitis/periodontitis, vascular dysfunction; (3) an increased presence of circulating cytokines; resulting in dysregulated hematological system, e.g., (4) amyloid plasma proteins and increased propensity for hypercoagulation (an important hallmark of systemic inflammation), hyperactivated platelets; endothelial dysfunction; (5) ultimately resulting in Parkinson's disease also being a true cardiovascular condition, where circulating inflammatory biomarkers (including bacterial inflammagens) may be used, not only as early detection of risk but also in tracking disease status such as factors IX and $\mathrm{X}$ and prothrombin [36, 37]. Therefore, the homeostatic control of the coagulation system/cascade can be disrupted when gingipains from $P$. gingivalis are present. Adams et al. [11] found, by using polyclonal antibodies, that gingipain R1 (RgpA) protease produced by $P$. gingivalis was present in platelet-poor plasma from PD patients. Furthermore, LPS from $P$. gingivalis caused hypercoagulability and RgpA hydrolyzed fibrin(ogen) so that healthy coagulation was inhibited. When RgpA and LPS were co-incubated, hyperclottable fibrin(ogen) could still be seen, supporting the findings that PD clots are dense and hyperclottable [38], and that a hyperclottable phenotype exists in PD patients. Whether the reducing effect of RgpA on clot formation, in terms of fibrinogen catalyzed by thrombin, also 


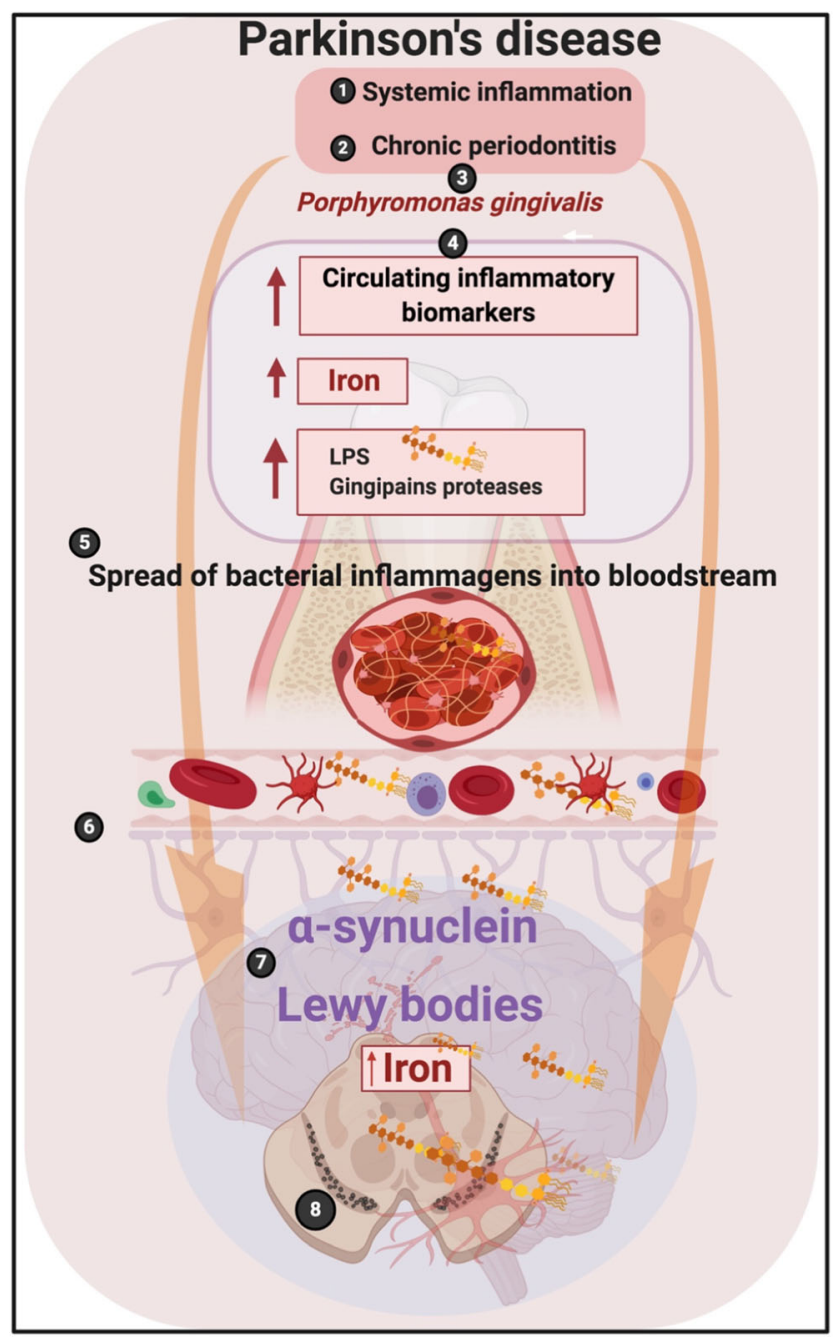

Fig. 2 Many individuals with Parkinson's disease suffer from both (1) systemic inflammation and (2) periodontitis. One of the bacteria that play a prominent role in the development of periodontitis is (3) $P$. gingivalis. (4) As a result of the systemic inflammation and periodontitis, there is an increase in circulating inflammatory biomarkers, including cytokines, iron, and bacterial inflammagens like LPS and proteases like gingipains. (5) The spread of these biomarkers via the bloodstream leads to a compromised blood-brain barrier (BBB) and an entry of particularly the bacterial inflammagens from $P$. gingivalis into the brain, where they may contribute to and fuel abnormal protein folding resulting in the formation of the abnormal presence of $\alpha$ Syn and Lewy body development, particularly in the dopaminergic neurons in the Parkinson's disease brain

occurs in blood plasma in vivo, where protease inhibitors and other RgpA targets exist, is unknown. However, circulating bacterial inflammagens such as LPS have also previously been found in PD [39-42] where they could be involved in both development and progression of the disease. This implies that bacteria might participate in PD as drivers of the disease through endotoxins and exotoxins acting as potent inducers of cytokines [43].

Intestinal bacteria may also be involved in PD where the role of the gut-brain-microbiota axis has been emphasized
[44-49]. However, this does not exclude involvement of oral bacteria since an oral-brain-microbiota axis may exist, as suggested recently for patients with autism spectrum disorder (ASD) [50]. Interestingly, intestinal dysbiosis has been associated with reduced LPS-binding protein in PD [51]. This protein may reverse the amyloid state of fibrin(ogen) [52]. Sampson et al. [53] reported that in mice, the gut microbiota was required for motor deficits, microglia activation, and $\alpha$ Syn pathology. Colonization of $\alpha$ Syn-overexpressing mice with microbiota from patients with PD increased physical impairments compared with microbiota transplants from healthy human donors.

\section{Other clinical studies supporting a potential periodontitis-Parkinson's disease association}

The study by Adams et al. [11] is not the only one reporting an association between periodontitis and PD. Chen et al. [54] found in a nationwide population-based case-control study that patients with periodontitis $(n=176)$ had a significantly higher risk of developing PD than controls $(n=275)$ with matching sex, age, index of year (occurrence of periodontitis), and comorbidity (adjusted hazard ratio $=1.431,95 \% \mathrm{Cl}$ [1.141-1.794], $p=0.002)$. In a similar study, Chen et al. [55] found that patients without periodontitis who underwent dental scaling over five consecutive years had a significantly lower risk of developing PD. Thus, dental scaling, which is the most common form of prophylaxis and treatment in periodontitis, significantly decreased the risk of developing PD. Other reports on an association between periodontitis and $\mathrm{PD}$ have come from Schwartz et al. [56], Zlotnik et al. [57], Kaur et al. [58], Hashioka et al. [59], and Olsen and Singhrao [60]. Although there are several studies indicating that periodontitis is more common in patients with PD, large longitudinal studies and randomized case-control or case-cohort studies are needed to substantiate this association [61].

\section{Concluding remarks}

The keystone pathogen of periodontitis, $P$. gingivalis, has been used in this paper as an example of a possible bacterial involvement in PD. The finding of R1 (Rgpa) and LPS, major inflammagens of $P$. gingivalis, in the circulation of a PD population, supports a role for $P$. gingivalis in the development of PD. This has been supported by several clinical studies. $P$. gingivalis cells in brain tissue have not yet been detected, so the clarification of this question will have to await further research. Even if the risk of periodontal disease for the development of PD and other neurological disorders needs to be better substantiated, that should not keep us from trying to 
prevent them by performing periodontitis prophylaxis through careful daily cleaning of teeth.

Funding information Open Access funding provided by University of Oslo (incl Oslo University Hospital). This collaboration received financial support from the Biotechnology and Biological Sciences Research Council (Grant BB/L025752/1) as well as the Medical Research Council of South Africa (MRC; Self-Initiated Research Program).

\section{Compliance with ethical standards}

Disclaimer The funders had no role in preparation of the manuscript. This is paper 26 in the series "a dormant blood microbiome in chronic, inflammatory diseases."

Open Access This article is licensed under a Creative Commons Attribution 4.0 International License, which permits use, sharing, adaptation, distribution and reproduction in any medium or format, as long as you give appropriate credit to the original author(s) and the source, provide a link to the Creative Commons licence, and indicate if changes were made. The images or other third party material in this article are included in the article's Creative Commons licence, unless indicated otherwise in a credit line to the material. If material is not included in the article's Creative Commons licence and your intended use is not permitted by statutory regulation or exceeds the permitted use, you will need to obtain permission directly from the copyright holder. To view a copy of this licence, visit http://creativecommons.org/licenses/by/4.0/.

\section{References}

1. Caton JG, Armitage G, Berglundh T, Chapple ILC, Jepsen S, Kornman KS, Mealey BL, Papapanou PN, Sanz M, Tonetti MS (2018) A new classification scheme for periodontal and periimplant diseases and conditions - introduction and key changes from the 1999 classification. J Clin Periodontol 45(Suppl 20):S1S8

2. Aas JA, Paster BJ, Stokes LN, Olsen I, Dewhirst FE (2005) Defining the normal bacterial flora of the oral cavity. J Clin Microbiol 43(11):5721-5732

3. Socransky SS (2000) Haffajee AD (2005) Periodontal microbial ecology. Periodontol 38:135-187

4. Lamont RJ, Koo H, Hajishengallis G (2018) The oral microbiota: dynamic communities and host interactions. Nat Rev Microbiol 16(12):745-759

5. Hajishengallis G, Darveau RP, Curtis MA (2012) The keystonepathogen hypothesis. Nat Rev Microbiol 10(10):717-725

6. Darveau RP, Hajishengallis G, Curtis MA (2012) Porphyromonas gingivalis as a potential community activist for disease. J Dent Res 91(9):816-820

7. Hajishengallis G, Lamont RJ (2012) Beyond the red complex and into more complexity: the polymicrobial synergy and dysbiosis (PSD) model of periodontal disease etiology. Mol Oral Microbiol 27(6):409-419

8. Duran-Pinedo AE, Chen T, Teles R, Starr JR, Wang X, Krishnan K, Frias-Lopez J (2014) Community-wide transcriptome of the oral microbiome in subjects with and without periodontitis. ISME $\mathbf{J}$ 8(8):1659-1672

9. Herrero ER, Fernandes S, Verspecht T, Ugarte-Berzal E, Boon N, Proost P, Bernaerts K, Quirynen M, Teughels W (2018) Dysbiotic biofilms deregulate the periodontal inflammatory response. J Dent Res 97(5):547-555
10. Darveau RP, Belton CM, Reife RA, Lamont RJ (1998) Local chemokine paralysis, a novel pathogenic mechanism for Porphyromonas gingivalis. Infect Immun 66(4):1660-1665

11. Adams B, Nunes JM, Page MJ, Roberts T, Carr J, Nell TA, Kell DB, Pretorius E (2019) Parkinson's disease: a systemic inflammatory disease accompanied by bacterial inflammagens. Front Aging Neurosci 11:210

12. More SV, Kumar H, Kim IS, Song S-Y, Choi D-K (2013) Cellular and molecular mediators of neuroinflammation in the pathogenesis of Parkinson's disease. Mediat Inflamm 2013:952375

13. Nolan YM, Sullivan AM, Toulouse A (2013) Parkinson's disease in the nuclear age of neuroinflammation. Trends Mol Med 19(3): 187-196

14. Taylor JM, Main BS, Crack PJ (2013) Neuroinflammation and oxidative stress: co-conspirators in the pathology of Parkinson's disease. Neurochem Int 62(5):803-819

15. Reale M, Iarlori C, Thomas A, Gambi D, Perfetti B, Di Nicola M, Onofrj M (2009) Peripheral cytokines profile in Parkinson's disease. Brain Behav Immun 23(1):55-63

16. Kannarkat GT, Boss JM, Tansey MG (2013) The role of innate and adaptive immunity in Parkinson's disease. J Park Dis 3(4):493-514

17. Brodacki B, Staszewski J, Toczyłowska B, Kozłowska E, Drela N, Chalimoniuk M, Stepien A (2008) Serum interleukin (IL-2, IL-10, IL-6, IL-4), TNF $\alpha$, and INFy concentrations are elevated in patients with atypical and idiopathic parkinsonism. Neurosci Lett 441(2): $158-162$

18. Qin X-Y, Zhang S-P, Cao C, Loh YP, Cheng Y (2016) Aberrations in peripheral inflammatory cytokine levels in Parkinson disease: a systematic review and meta-analysis. JAMA Neurol 73(11):13161324

19. Lotankar S, Prabhavalkar KS, Bhatt LK (2017) Biomarkers for Parkinson's disease: recent advancement. Neurosci Bull 33(5): 585-597

20. Kell DB, Pretorius E (2015) On the translocation of bacteria and their lipopolysaccharides between blood and peripheral locations in chronic, inflammatory diseases: the central roles of LPS and LPSinduced cell death. Integr Biol (Camb) 7(11):1339-1377

21. Kell DB, Pretorius E (2018) No effects without causes: the iron dysregulation and dormant microbes hypothesis for chronic, inflammatory diseases. Biol Rev Camb Philos Soc 93(3):1518-1557

22. Olumuyiwa-Akeredolu O-O, Page MJ, Soma P, Pretorius E (2019) Platelets: emerging facilitators of cellular crosstalk in rheumatoid arthritis. Nat Rev Rheumatol 15(4):237-248

23. Olsen I (2015) From the Acta Prize Lecture 2014: the periodontalsystemic connection seen from a microbiological standpoint. Acta Odontol Scand 73(8):563-568

24. Olsen I, Singhrao SK (2015) Can oral infection be a risk factor for Alzheimer's disease? J Oral Microbiol 7:29143

25. Poole S, Singhrao SK, Chukkapalli S, Rivera M, Velsko I, Kesavalu L, Crean SJ (2015) Active invasion of Porphyromonas gingivalis and infection-induced complement activation in $\mathrm{ApoE}^{-/-}$ mice brains. J Alzheimers Dis 43(1):67-80

26. Dominy SS, Lynch C, Ermini F, Benedyk M, Marczyk A, Konradi A, Nguyen M, Haditsch U, Raha D, Griffin C, Holsinger LJ, Arastu-Kapur S, Kaba S, Lee A, Ryder MI, Potempa B, Mydel P, Hellvard A, Adamowicz K, Hasturk H, Walker GD, Reynolds EC, Faull RLM, Curtis MA, Dragunow M, Potempa J (2019) Porphyromonas gingivalis in Alzheimer's disease brains: evidence for disease causation and treatment with small-molecule inhibitors. Sci Adv 5(1):eaau3333

27. Guo Y, Nguyen K-A, Potempa J (2010) Dichotomy of gingipains action as virulence factors: from cleaving substrates with the precision of a surgeon's knife to a meat chopper-like brutal degradation of proteins. Periodontol 2000 54(1):15-44

28. Slaney JM, Curtis MA (2008) Mechanisms of evasion of complement by Porphyromonas gingivalis. Front Biosci 13:188-196 
29. Olsen I, Singhrao SK (2020) Is there a link between genetic defects in the complement cascade and Porphyromonas gingivalis in Alzheimer's disease? J Oral Microbiol 12(1):1676486

30. Wong KT, Groove JS, Grandinetti A, Curb JD, Yee M, Blanchette P, Ross GW, Rodriguez BI (2010) Association of fibrinogen with Parkinson disease in elderly Japanese-American men: a prospective study. Neuroepidemiology 34(1):50-54

31. Ton GN, Jain S, Biggs ML, Thacker EL, Strotmeyer ES, Boudreau R, Newman AB, Longstreth WT Jr, Checkoway H (2012) Markers of inflammation in prevalent and incident Parkinson's disease in the cardiovascular health study. Parkinsonism Relat Disord 18(3):274 278

32. Pretorius E, Mbotwe S, Kell DB (2017) Lipopolysaccharidebinding protein (LBP) reverses the amyloid state of fibrin seen in plasma of type 2 diabetics with cardiovascular co-morbidities. Sci Rep 7(1):9680

33. Pretorius E, Page MJ, Engelbrecht L, Ellis GC, Kell DB (2017) Substantial fibrin amyloidgenesis in type 2 diabetes assessed using amyloid-selective fluorescent stains. Cardiovasc Diabetol 16(1): 141

34. Pretorius E, Bester J, Page MJ, Kell DB (2018) The potential of LPS-binding protein to reverse amyloid formation in plasma fibrin individuals with Alzheimer-type dementia. Front Aging Neurosci $10: 257$

35. Imamura T, Potempa J, Pike RN, Moore JN, Barton MH, Travis J (1995) Effect of free and vesicle-bound cysteine proteinases of Porphyromonas gingivalis on plasma clot formation: implications for bleeding tendency at periodontitis sites. Infect Immun 63(12): 4877-4882

36. Imamura T, Potempa J, Tanase S, Travis J (1997) Activation of blood coagulation factor $\mathrm{X}$ by arginine-specific cysteine proteinases (gingipain-PRs) from Porphyromonas gingivalis. J Biol Chem 172(25):16062-16067

37. Imamura T, Banbula A, Pereira PJ, Travis J, Potempa J (2001) Activation of human prothrombin by arginine-specifc cysteine proteinases (gingipains R) from Porphyromonas gingivalis. J Biol Chem 276(22):18984-18991

38. Pretorius E, Page MJ, Mbotwe S, Kell DB (2018) Lipopolysaccharide-binding protein (LBP) can reverse the amyloid state of fibrin seen or induced in Parkinson's disease: implications. PLoS One 13(3):e0192121

39. Tufekci KU, Genc S, Genc K (2011) The endotoxin-induced neuroinflammation model of Parkinson's disease. Parkinson's Dis 2011:487450

40. De Chiara G, Marcocci ME, Sgarbanti R, Civitelli L, Ripoli C, Piacentini R, Garaci E, Grassi C, Palamara AT (2012) Infectious agents and neurodegeneration. Mol Neurobiol 46(3):614-638

41. Potgieter M, Bester J, Kell DB, Pretorius E (2015) The dormant blood microbiome in chronic, inflammatory diseases. FEMS Microbiol Rev 39(4):567-591

42. Friedland RP, Chapman MR (2017) The role of microbial amyloid in neurodegeneration. PLoS Pathog 13(12):e1006654

43. Cavaillon J-M (2018) Exotoxins and endotoxins: inducers of inflammatory cytokines. Toxicon 149:45-53

44. Mulak A, Bonaz B (2015) Brain-gut-microbiota axis in Parkinson's disease. World J Gastroenterol 21(37):10609-10620

45. Scheperjans F, Derkinderen P, Borghammer P (2018) The gut and Parkinson's disease: hype or hope? J Park Dis 8(s1):S31-S39

46. Caputi V, Giron MC (2018) Microbiome-gut-brain axis and Tolllike receptors in Parkinson's disease. Int J Mol Sci 19(6):1689
47. Sun M-F, Shen Y-Q (2018) Dysbiosis of gut microbiota and microbial metabolites in Parkinson's disease. Ageing Res Rev 45:53-61

48. Sun M-F, Zhu Y-L, Zhou Z-L, Jia X-B, Xu Y-D, Yang Q, Cui C, Shen Y-Q (2018) Neuroprotective effects of fecal microbiota transplantation on MPTP-induced Parkinson's disease mice: gut microbiota, glial reaction and TLR4/TNF- $\alpha$ signaling pathway. Brain Behav Immun 70:48-60

49. Yang D, Zhao D, Shah SZA, Wu W, Lai M, Zhang X, Li J, Guan Z, Zhao H, Li W, Gao H, Zhou X, Yang L (2019) The role of the gut microbiota in the pathogenesis of Parkinson's disease. Front Neurol 10:1155

50. Olsen I, Hicks SD (2019) Oral microbiota and autism spectrum disorder (ASD). J Oral Microbiol 12(1):1702806

51. Hasegawa S, Goto S, Tsuji H, Okuno T, Asahara T, Nomoto K, Shibata A, Fujisawa Y, Minato T, Okamoto A, Ohno K, Hirayama M (2015) Intestinal dysbiosis and lowered serum lipopolysaccharide-binding protein in Parkinson's disease. PLoS One 10(11):e0142164

52. Pretorius E, Page MJ, Mbotwe S, Kell DB (2018) Lipopolysaccharide-binding protein (LBP) can reverse the amyloid state of fibrin seen or induced in Parkinson's disease. PLoS One 13(3): $\mathrm{e} 0192121$

53. Sampson TR, Debelius JW, Thron T, Janssen S, Shastri GG, Ilhan ZE, Challis C, Schretter CE, Rocha S, Gradinaru V, Chesselet M-F, Keshavarzian A, Shannon KM, Krajmalnik-Brown R, WittungStafshede P, Knight R, Mazmanian SK (2016) Gut microbiota regulate motor deficits and neuroinflammation in a model of Parkinson's disease. Cell 167(6):1469-1480.e12

54. Chen C-K, Huang J-Y, Wu Y-T, Chang Y-C (2017) Periodontal inflammatory disease is associated with the risk of Parkinson's disease: a population-based retrospective matched-cohort study. PeerJ 5:e3647

55. Chen C-K, Huang J-Y, Wu Y-T, Chang Y-C (2018) Dental scaling decreases the risk of Parkinson's disease: a nationwide populationbased nested case-control study. Int J Environ Res Public Health 15(8): 1587

56. Schwarz J, Heimhilger E, Storch A (2006) Increased periodontal pathology in Parkinson's disease. J Neurol 253(5):608-611

57. Zlotnik Y, Balash Y, Korczyn AD, Giladi N, Gurevich T (2015) Disorders of the oral cavity in Parkinson's disease and parkinsonian syndromes. Parkinsons Dis 2015:379482

58. Kaur T, Uppoor A, Naik D (2016) Parkinson's disease and periodontitis - the missing link? A review. Gerodontology 33(4):434 438

59. Hashioka S, Inoue K, Miyaoka T, Hayashida M, Wake R, Oh-Nishi A, Inagaki M (2019) The possible causal link of periodontitis to neuropsychiatric disorders: more than psychosocial mechanisms. Int J Mol Sci 20(15):3723

60. Olsen I, Singhrao SK (2019) Poor oral health and its neurological consequences: mechanisms of Porphyromonas gingivalis involvement in cognitive dysfunction. Curr Oral Health Rep 6:120-129

61. Holmstrup P, Damgaard C, Olsen I, Klinge B, Flyvbjerg A, Nielsen $\mathrm{CH}$, Hansen PR (2017) Comorbidity of periodontal disease: two sides of the same coin? An introduction for the clinician. J Oral Microbiol 9(1):1332710

Publisher's note Springer Nature remains neutral with regard to jurisdictional claims in published maps and institutional affiliations. 